\title{
Existence of solutions of non-autonomous fractional differential equations with integral impulse condition
}

\author{
Ashish Kumar' ${ }^{1}$, Harsh Vardhan Singh Chauhan², Chokkalingam Ravichandran ${ }^{3} \mathbb{D}$, \\ Kottakkaran Sooppy Nisar ${ }^{4 *}$ (D) and Dumitru Baleanu ${ }^{5,6,7}$
}

\author{
"Correspondence: \\ n.sooppy@psau.edu.sa \\ ${ }^{4}$ Department of Mathematics, \\ College of Arts and Sciences, Prince \\ Sattam bin Abdulaziz University, \\ Wadi Aldawasir 11991, Saudi Arabia \\ Full list of author information is \\ available at the end of the article
}

\begin{abstract}
In this paper, we investigate the existence of solution of non-autonomous fractional differential equations with integral impulse condition by the measure of non-compactness (MNC), fixed point theorems, and k-set contraction. The obtained results are verified via a supporting example.
\end{abstract}

MSC: $34 \mathrm{~K} 37 ; 34 \mathrm{~K} 35 ; 47 \mathrm{H} 10$

Keywords: Functional-differential equations with fractional derivatives; Non-autonomous evolution equation; Fixed point theorems

\section{Introduction}

Fractional calculus is a generalization of the standard integer calculus. The advantage of fractional calculus over integer-order calculus is that it provides a great deal for the kind of thought and hereditary characteristics of diversified materials and methods. From the past two decades, fractional calculus has attracted research attention towards itself due to its importance in several parts of science, like physics, fluid mechanics, heat conduction $[1,19,21,24,26,27,32,33,37-42]$. We can relate to the monographs $[2,22,30,36]$ for the fundamentals and to $[4,44,45]$ for the current developments in the field of fractional calculus.

In recent years, non-autonomous differential equations of integer order, as well as fractional order, have been studied by many researchers. One can see the references [5-8, 10$13,15-17,20,25,35]$ for more details. In [14], Chen et al. discussed the existence of mild solutions as well as approximate controllability for a class of non-autonomous evolution systems of parabolic type with nonlocal conditions in Banach spaces by using the Schauder's fixed-point theorem as well as the theory of an evolution family. In the same year, Chen et al. [9] explored the existence of mild solutions for the initial value problem to a new class of abstract evolution equations with non-instantaneous impulses on ordered Banach spaces by using a perturbation technique and by dropping the compactness condition on the semigroup. Malik et al. [28] used the Rothe's fixed point theorem to study the

(c) The Author(s) 2020. This article is licensed under a Creative Commons Attribution 4.0 International License, which permits use, sharing, adaptation, distribution and reproduction in any medium or format, as long as you give appropriate credit to the original author(s) and the source, provide a link to the Creative Commons licence, and indicate if changes were made. The images or other third party material in this article are included in the article's Creative Commons licence, unless indicated otherwise in a credit line to the material. If material is not included in the article's Creative Commons licence and your intended use is not permitted by statutory regulation or exceeds the permitted use, you will need to obtain permission directly from the copyright holder. To view a copy of this licence, visit http://creativecommons.org/licenses/by/4.0/. 
controllability of non-autonomous nonlinear differential system with non-instantaneous impulses in the space $\mathbb{R}^{n}$. By using Krasnoselskii's fixed point theorem, Wang et al. [43] formed a set of sufficient conditions for the existence and stability for a class of impulsive non-autonomous differential equations. Kucche [23] investigated the existence and uniqueness of mild solutions for impulsive delay integrodifferential equations with integral impulses in Banach spaces by using Krasnoselskii-Schaefer fixed point theorem.

Motivated by the above, we discuss the non-autonomous fractional differential system with integral impulses in the following form:

$$
\begin{aligned}
& { }^{C} D^{q} u(s)+\mathbb{A}(s) u(s)=f(s, u(s)), \\
& u(0)=u_{0}, \\
& \Delta u\left(s_{k}\right)=I_{k}\left(\int_{s_{k}-\tau_{k}}^{s_{k}-\theta_{k}}(G, u(t)) d t\right),
\end{aligned}
$$

where ${ }^{C} D^{q}$ denotes the CFD of order $0<q \leq 1, J=[0, S], S \in \mathbb{R}$ is a positive constant. Suppose that $F$ is a Banach space and $\{\mathbb{A}(s)\}_{s \in J}$ is a family of closed linear operator from $F$ to $F$. The domain of $\{\mathbb{A}(s)\}$ is $D(\mathbb{A})$ which is independent of $s$ and dense in $F ; f: J \times F \rightarrow F$ is continuous and with $G: J \times F \rightarrow F$ are given functions; $0=s_{0}<s_{1}<\cdots<s_{m}<s_{m+1}=$ $S, I_{k} \in C(F, F)$ are bounded functions, $0 \leq \theta_{k} \leq \tau_{k} \leq s_{k}-s_{k-1}$ for $k=1,2, \ldots, m, \Delta u(s)=$ $u\left(s_{k}^{+}\right)-u\left(s_{k}^{-}\right), u\left(s_{k}^{+}\right)=\lim _{\epsilon \rightarrow 0} u\left(s_{k}+\epsilon\right), u\left(s_{k}^{-}\right)=\lim _{\epsilon \rightarrow 0} u\left(s_{k}-\epsilon\right)$.

\section{Preliminaries}

Let $F$ be a Banach space with norm $\|\cdot\|$. Further $C(J, F)=\{u: u: J \rightarrow F$ is continuous $\}$ denotes a Banach space with norm $\|u\|_{C}=\sup _{s \in J}\|u(s)\|$ and $\mathcal{L}(F)$ denotes the Banach space of all bounded linear operators in $F$ with the operator norm topology. Let $L^{1}(J, F)$ be the Banach space of all Bochner integrable functions with the norm $\|u\|_{1}=\int_{0}^{S}\|u(s)\| d s$.

Now, we recall some definitions of fractional derivatives and integral.

Definition 2.1 ([22]) The fractional integral operator (in Riemann-Liouville sense) of order $q>0$ of a function $u$ is defined as

$$
I^{q} u(s)=\frac{1}{\Gamma(q)} \int_{0}^{s}(s-t)^{q-1} u(t) d t,
$$

here $\Gamma(\cdot)$ denotes the Euler Gamma function.

Definition 2.2 ([22]) We define the derivative of $u$ of the fractional order $q>0$ in the Caputo sense as

$$
{ }^{C} D^{q} u(s)=\frac{1}{\Gamma(1-q)} \int_{0}^{s}(s-t)^{-q} u^{\prime}(t) d t,
$$

here $0<q \leq 1$ and $u^{\prime}(t)=\frac{d u(t)}{d t}$.

A measurable function $f:[0, \infty) \rightarrow F$ is called Bochner integrable if $\|f\|$ is Lebesgue integrable. The integrals which appear in (2.1) and (2.2) are taken in Bochner's sense. Let the operator $-\mathbb{A}(s)$ satisfies the following conditions: 
$\left(H_{1}\right) \mathbb{A}(s)$ is a closed operator, the domain of $\mathbb{A}(s)$ is independent of $s$, and dense in $F$.

$\left(H_{2}\right)$ For any $\lambda \geq 0$, the operator $\lambda I+\mathbb{A}(s)$ has a bounded inverse operator $[\lambda I+\mathbb{A}(s)]^{-1}$ in $\mathcal{L}(F)$ and

$$
\left\|[\lambda I+\mathbb{A}(s)]^{-1}\right\| \leq \frac{C}{|\lambda|+1},
$$

where $C$ is a positive constant independent of $s$ and $\lambda$.

$\left(H_{3}\right)$ For any $s, \tau, t \in J$, there is a constant $p \in(0,1]$ such that

$$
\left\|[\mathbb{A}(s)-\mathbb{A}(\tau)] \mathbb{A}^{-1}(t)\right\| \leq C|s-\tau|^{p},
$$

where the constants $p$ and $C>0$ are independent of $s, \tau$, and $t$.

Following Pazy [34], $\left(H_{1}\right)$ means that for each $t \in J,-A(t)$ generates an analytic semigroup $e^{-s \mathbb{A}(t)}(s>0)$, and there is a $C>0$ independent of both $s$ and $t$ such that $\left\|\mathbb{A}^{n}(t) e^{-s \mathbb{A}(t)}\right\| \leq \frac{C}{s^{n}}$, where $n=0,1, s>0, t \in J$.

By [18], we can give the definition of operators $\tilde{\Psi}(s, t), \tilde{\Phi}(s, \sigma)$, and $\tilde{U}(s)$ :

$$
\begin{aligned}
& \tilde{\Psi}(s, t)=q \int_{0}^{\infty} \theta s^{q-1} \xi_{q} e^{-s^{q} \theta \mathbb{A}(t)} d \theta, \\
& \tilde{\Phi}(s, \sigma)=\sum_{k=1}^{\infty} \tilde{\Phi}_{k}(s, \sigma), \\
& \tilde{U}(s)=-\mathbb{A}(s) \mathbb{A}^{-1}(0)-\int_{0}^{s} \tilde{\Phi}(s, t) \mathbb{A}(t) \mathbb{A}^{-1}(0) d t,
\end{aligned}
$$

where $\xi_{q}$ is probability density function defined on $[0, \infty)$ such that it's Laplace transform is given by

$$
\begin{aligned}
& \int_{0}^{\infty} e^{-\theta x} \xi_{q}(\theta) d \theta=\sum_{i=0}^{\infty} \frac{(-x)^{i}}{\Gamma(1+q i)}, \quad 0<q \leq 1, z>0, \\
& \tilde{\Phi}_{1}(s, \sigma)=[\mathbb{A}(s)-\mathbb{A}(\sigma)] \tilde{\Psi}(s-\sigma, \sigma), \\
& \tilde{\Phi}_{k+1}(s, \sigma)=\int_{\sigma}^{s} \tilde{\Phi}_{k}(s, t) \tilde{\Phi}_{1}(t, \sigma) d \sigma, \quad k=1,2, \ldots .
\end{aligned}
$$

Using the above facts, we define the mild solution of problem (1.1)-(1.3).

Definition 2.3 ([29]) A function $u \in C(J, F)$ is called a mild solution of (1.1)-(1.3) if it satisfies the integral equation

$$
\begin{aligned}
u(s)= & u_{0}+\int_{0}^{s} \tilde{\Psi}(s-\sigma, \sigma) \tilde{U}(\sigma) \mathbb{A}(0) u_{0} d \sigma \\
& +\int_{0}^{s} \tilde{\Psi}(s-\sigma, \sigma) f(\sigma, u(\sigma)) d \sigma \\
& +\int_{0}^{s} \int_{0}^{\sigma} \tilde{\Psi}(s-\sigma, \sigma) \tilde{\Phi}(\sigma, t) f(t, u(t)) d t d \sigma \\
& +\sum_{0<s_{k}<s} \tilde{\Psi}\left(s_{k}-\sigma, \sigma\right) I_{k}\left(\int_{s_{k}-\tau_{k}}^{s_{k}-\theta_{k}} G(t, u(t)) d t\right) .
\end{aligned}
$$


The following lemma gives some properties of $\tilde{\Psi}(s, t), \tilde{\Phi}(s, \sigma)$, and $\tilde{U}(s)$ that are required to prove our main result.

Lemma 2.1 ([18]) Functions $\tilde{\Psi}(s-\sigma, \sigma)$ and $\mathbb{A}(s) \tilde{\Psi}(s-\sigma, \sigma)$ are continuous in the uniform topology in $s$ and $\sigma$, where $s \in J, 0 \leq \sigma \leq s-\epsilon$ for any $\epsilon>0$ and

$$
\|\tilde{\Psi}(s-\sigma, \sigma)\| \leq(s-\sigma)^{q-1},
$$

where $C>0$ is independent of both $s$ and $\sigma$. Moreover,

$$
\|\tilde{\Phi}(s, \sigma)\| \leq(s-\sigma)^{p-1}
$$

and

$$
\|\tilde{U}(s)\| \leq C\left(1+t^{p}\right) .
$$

Next, we define the MNC, which is required in our results.

Definition 2.4 ([3]) Assume that $G$ is a bounded set of $F$. Then the Kuratowski MNC $\mu(\cdot)$ defined on $G$ is

$$
\mu(G)=\inf \left\{\delta>0: G=\bigcup_{k=1}^{n} G_{k} \text { and } \operatorname{diam}\left(G_{k}\right) \leq \delta \text { for } k=1,2, \ldots, n\right\} .
$$

Some properties of $\mu(\cdot)$ are given in the following lemma.

Lemma 2.2 ([3]) Let $Z, W$ be bounded subsets of $F$ and $\lambda \in \mathbb{R}$. Then

1. $\mu(Z)=0$ if and only if $\bar{Z}$ is compact, where $\bar{Z}$ denotes the closure of $Z$;

2. $Z \subseteq W$ implies $\mu(Z) \leq \mu(W)$;

3. $\mu(\bar{Z})=\mu(\operatorname{conv} Z)=\mu(Z)$, where conv $Z$ denotes the convex hull of $Z$;

4. $\mu(Z \cup W)=\max \{\mu(Z), \mu(W)\}$;

5. $\mu(\lambda Z)=|\lambda| \mu(Z)$, where $\lambda Z=\{\lambda z: z \in Z\}$;

6. $\mu(Z+W) \leq \mu(Z)+\mu(W)$, where $Z+W=\{z+w: z \in Z, w \in W\}$;

7. If $G$ is any bounded subset of $D(P)$, and if $P: D(P) \subset F \rightarrow F$ is a Lipschitz-continuous mapping with constant $k$, then $\mu(P(G)) \leq k \mu(G)$.

In this article, $\mathrm{MNC}$ on the set $F$ and $C(J, F)$ is denoted by $\mu(\cdot)$ and $\mu_{C}(\cdot)$, respectively. For any $s \in J$ and $B \subset C(J, F)$, we denote $B(s)=\{u(s): u \in B\}$, then $B(s) \subset F$. The boundedness of $B(s) \subset C(J, F)$ implies the boundedness of $B(s)$ in $F$ and $\mu(B(s)) \leq \mu_{C}(B)$.

The prove our main result, the following lemmas are required.

Lemma 2.3 ([3]) Assume that $B$ is a bounded subset of $F$. Then there is a countable subset $B_{0}$ of $B$ such that $\mu(B) \leq 2 \mu\left(B_{0}\right)$.

Lemma 2.4 ([31]) If $B=\left\{u_{n}\right\}_{1}^{\infty} \subset C([0, S], F)$ is a countable set in a Banach space $F$ and there is a function $m \in L^{1}\left([0, S], \mathbb{R}^{+}\right)$such that for every $n \in \mathbb{N}$,

$$
\left\|u_{n}(s)\right\| \leq m(s), \quad \text { a.e. } s \in[0, S]
$$


then $\mu(B(s))$ is Lebesgue integrable on $[0, S]$ and

$$
\mu\left(\left\{\int_{0}^{s} \mu_{n}(s) d s: n \in \mathbb{N}\right\}\right) \leq 2 \int_{0}^{s} \mu(B(s)) d s
$$

Lemma 2.5 ([2]) If $B \subset C(J, F)$ is equicontinuous and bounded in a Banach space $F$ then $\mu(B(s))$ is continuous on $[0, S]$ and $\mu_{C}(B)=\max _{s \in[0, S]} \mu(B(s))$.

Definition 2.5 ([3]) If $G$ is a nonempty subset of $F$, then $P: G \rightarrow F$ is called to $k$-set contractive if it is continuous and there is a constant $k \in[0,1)$ such that for every bounded subset $D$ of $G$, we have

$$
q(P(D)) \leq k q(D)
$$

Lemma 2.6 ([3]) Assuming that $D$ is a closed, bounded, and convex subset of $F, P: D \rightarrow D$ has at least one fixed point in $D$ if it is $k$-set contractive.

\section{Main results}

$\left(F_{1}\right)$ For $r>0$ there are constants $0<q_{1}<\min (q, p), \rho>0$ and a function $h_{r} \in L^{\frac{1}{q_{1}}}\left(J, \mathbb{R}^{+}\right)$ such that for any $s \in J$ and $u \in F$ with $\|u\| \leq r,\|f(s, u)\| \leq h_{r}(s)$ and

$$
\lim _{r \rightarrow \infty} \inf \frac{\left\|h_{r}\right\|_{L^{\frac{1}{q_{1}}}}[0, S]}{r}=\rho \leq \infty
$$

$\left(F_{2}\right)$ There is an $L>0$ such that for every bounded, countable, and equicontinuous set $D \subset F$

$$
\mu(f(s, D)) \leq L \mu(D) \quad \text { for all } s \in J
$$

$\left(F_{3}\right)$ There are constants $L_{N}, M$ such that

$$
\begin{aligned}
& \left\|N\left(s, v_{1}\right)-N\left(s, v_{2}\right)\right\| \leq L_{N}\left\|v_{1}-v_{2}\right\|, \\
& M=\sup _{s \in[0, S]}\|N(s, 0)\| ;
\end{aligned}
$$

$\left(F_{4}\right)$ There are constants $D_{k}(k=1,2,3, \ldots, m)$ such that

$$
\left\|I_{k}\left(w_{1}\right)-I_{k}\left(w_{2}\right)\right\| \leq D_{k}\left\|w_{1}-w_{2}\right\| ; \quad k=1,2, \ldots, m \text { for } w_{1}, w_{2} \in F
$$

In this study, $\mathbf{B}(q, p)=\int_{0}^{1} s^{q-1}(1-s)^{p-1} d s$ is the Beta function.

Theorem 3.1 Iff $J \times F \rightarrow F$ is continuous and satisfies conditions $\left(F_{1}\right)-\left(F_{4}\right)$ then there is at least one mild solution of (1.1)-(1.3) in $C(J, F)$ provided that

$$
\frac{C S^{q-q_{1}} \rho}{\left(1-C^{*} S^{q+1}\right)}\left[\left(\frac{1-q_{1}}{q-q^{1}}\right)^{1-q_{1}}+C \mathbf{B}(q, p) S^{p}\left(\frac{1-q_{1}}{q+p-q_{1}}\right)^{1-q_{1}}\right]<1,
$$


and

$$
C S^{q}\left[\frac{L_{1}}{q}+\frac{2 C L \mathbf{B}(q, p) S^{p}}{q+p}+2 L_{N} S\right] \leq \frac{1}{4}
$$

Proof: Let the operator $\mathcal{P}: C(J, F) \rightarrow C(J, F)$ be defined by

$$
\begin{aligned}
(\mathcal{P} u)(s)= & u_{0}+\int_{0}^{s} \tilde{\Psi}(s-\sigma, \sigma) \tilde{U}(n) \mathbb{A}(0) u_{0} d \sigma \\
& +\int_{0}^{s} \tilde{\Psi}(s-\sigma, \sigma) f(\sigma, u(\sigma)) d \sigma \\
& +\int_{0}^{s} \int_{0}^{\sigma} \tilde{\Psi}(s-\sigma, \sigma) \tilde{\Phi}(\sigma, t) f(t, u(t)) d t d \sigma \\
& +\sum_{0<s_{k}<s} \tilde{\Psi}\left(s_{k}-\sigma, \sigma\right) I_{k}\left(\int_{s_{k}-\tau_{k}}^{s_{k}-\theta_{k}} N(t, u(t)) d t\right) .
\end{aligned}
$$

First, we show that $\mathcal{P}$ maps $G_{R}$ to $G_{R}$ which is a bounded, closed and convex set, where $R$ is a positive constant such that $G_{R}=\{u \in C(J, F):\|u(s)\| \leq R$ for $\forall s \in J\}$. If this were not true, then there would exist $s^{r} \in J$ and $u^{r} \in G_{R}$ such that $\left\|\left(\mathcal{P} u^{r}\right)\left(s^{r}\right)\right\|>r$ for each $r>0$. Now by using Hölder inequality, $\left(F_{1}\right)$ and Lemma 2.1, we get

$$
\begin{aligned}
& r<\left\|\left(\mathcal{P} u^{r}\right)\left(s^{r}\right)\right\| \\
& \leq\left\|u_{0}\right\|+\left\|\int_{0}^{s^{r}} \tilde{\Psi}\left(s^{r}-\sigma, \sigma\right) \tilde{U}(\sigma) \mathbb{A}(0) u_{0} d \sigma\right\| \\
& +\left\|\int_{0}^{s^{r}} \tilde{\Psi}\left(s^{r}-\sigma, \sigma\right) f\left(\sigma, u^{r}(\sigma)\right) d \sigma\right\| \\
& +\left\|\int_{0}^{s^{r}} \int_{0}^{\sigma} \tilde{\Psi}\left(s^{r}-\sigma, \sigma\right) \tilde{\Phi}(\sigma, t) f\left(t, u^{r}(t)\right) d t d \sigma\right\| \\
& +\left\|\sum_{0<s_{k}<s} \tilde{\Psi}\left(s_{k}^{r}-\sigma, \sigma\right) I_{k}\left(\int_{s_{k}-\tau_{k}}^{s_{k}-\theta_{k}} N\left(t, u^{r}(t)\right) d t\right)\right\| \\
& \leq\left\|u_{0}\right\|+C^{2}\left\|\int_{0}^{s^{r}}\left(s^{r}-\sigma\right)^{q-1}\left(1+\sigma^{p}\right) d \sigma\right\|\left\|\mathbb{A}(0) u_{0}\right\| \\
& +C \int_{0}^{s^{r}}\left(s^{r}-\sigma\right)^{q-1} h_{r}(\sigma) d \sigma \\
& +C^{2} \int_{0}^{s^{r}} \int_{0}^{\sigma}\left(s^{r}-\sigma\right)^{q-1}(\sigma-t)^{p-1} h_{r}(t) d t d \sigma \\
& +\sum_{0<s_{k}<s} C L_{N} D_{k}\left(s_{k}^{r}-\sigma\right)^{q-1}\left(\int_{s_{k}-\tau_{k}}^{s_{k}-\theta_{k}}\left[\left\|u^{r}\right\|+\|N(t, 0)\|\right] d t\right) \\
& \leq\left\|u_{0}\right\|+C^{2}\left(s^{r}\right)^{q}\left[\frac{1}{q}+\left(s^{r}\right)^{p} B(q, p+1)\right]\left\|\mathbb{A}(0) u_{0}\right\| \\
& +C \int_{0}^{s^{r}}\left(s^{r}-\sigma\right)^{q-1} h_{r}(\sigma) d \sigma+C^{2} \mathbf{B}(q, p) \int_{0}^{s^{r}}\left(s^{r}-\sigma\right)^{q+p-1} h_{\sigma}(t) d t d \sigma \\
& +\sum_{0<s_{k}<s} C L_{N} D_{k} S^{p}(r+M) 2 S
\end{aligned}
$$




$$
\begin{aligned}
\leq & \left\|u_{0}\right\|+C^{2} S^{q}\left[\frac{1}{q}+(S)^{p} B(q, p+1)\right]\left\|\mathbb{A}(0) u_{0}\right\| \\
& +C\left(\int_{0}^{s^{r}}\left(s^{r}-\sigma\right)^{\frac{q-1}{1-q_{1}}} d \sigma\right)^{1-q}\left(\int_{0}^{s^{r}} h_{r}^{\frac{1}{q_{1}}}(\sigma) d \sigma\right)^{q_{1}} \\
& +C^{2} \mathbf{B}(q, p)\left(\int_{0}^{s^{r}}\left(s^{r}-\sigma\right)^{\frac{q+p-1}{1-q_{1}}} d \sigma\right)^{1-q}\left(\int_{0}^{s^{r}} h_{r}^{\frac{1}{q_{1}}}(\sigma) d \sigma\right)^{q_{1}} \\
& +\sum_{0<s_{k}<s} 2 C L_{N} D_{k} S^{q+1}(r+M) \\
\leq & \left\|u_{0}\right\|+C^{2} S^{q}\left[\frac{1}{q}+(S)^{p} B(q, p+1)\right]\left\|\mathbb{A}(0) u_{0}\right\| \\
& +C S^{q-q_{1}}\left(\frac{1-q_{1}}{q-q^{1}}\right)^{1-q_{1}}\left\|h_{r}\right\|_{L^{\frac{1}{q_{1}}}}[0, S] \\
& +C^{2} \mathbf{B}(q, p) S^{q+p-q_{1}}\left(\frac{1-q_{1}}{q+p-q_{1}}\right)^{1-q_{1}}\left\|h_{r}\right\|_{L^{\frac{1}{q_{1}}}[0, S]} \\
& +r C^{*} S^{q+1}+M C^{*} S^{q+1},
\end{aligned}
$$

which gives

$$
\begin{aligned}
r\left(1-C^{*} S^{q+1}\right) \leq & \left\|u_{0}\right\|+C^{2} S^{q}\left[\frac{1}{q}+(S)^{p} B(q, p+1)\right]\left\|\mathbb{A}(0) u_{0}\right\| \\
& +C S^{q-q_{1}}\left(\frac{1-q_{1}}{q-q^{1}}\right)^{1-q_{1}}\left\|h_{r}\right\|_{L^{\frac{1}{q_{1}}}[0, S]} \\
& +C^{2} \mathbf{B}(q, p) S^{q+p-q_{1}}\left(\frac{1-q_{1}}{q+p-q_{1}}\right)^{1-q_{1}}\left\|h_{r}\right\|_{L^{\frac{1}{q_{1}}}[0, S]} \\
& +M C^{*} S^{q+1} .
\end{aligned}
$$

Dividing both sides of (3.6) by $r\left(1-C^{*} S^{q+1}\right)$, using $\left(F_{1}\right)$, and taking the limit as $r \rightarrow \infty$, we get

$$
1 \leq \frac{C S^{q-q_{1}} \rho}{\left(1-C^{*} S^{q+1}\right)}\left[\left(\frac{1-q_{1}}{q-q^{1}}\right)^{1-q_{1}}+C \mathbf{B}(q, p) S^{p}\left(\frac{1-q_{1}}{q+p-q_{1}}\right)^{1-q_{1}}\right]<1,
$$

which is a contradiction. Therefore $\mathcal{P}: G_{R} \rightarrow G_{R}$.

Now, we prove that $\mathcal{P}: G_{R} \rightarrow G_{R}$ is a continuous operator. Consider $\left\{u_{n}\right\}_{n=1}^{\infty} \subset G_{R}$ such that $\lim _{n \rightarrow \infty} u_{n}=u$ in $G_{R}$. Since the function $f$ is continuous in the second variable, for any $s \in J$, we get

$$
\lim _{n \rightarrow \infty}\left\|f\left(s, u_{n}(s)\right)-f(s, u(s))\right\|=0 .
$$

From (3.5) and Lemma 2.1, we get

$$
\begin{aligned}
\left\|\left(\mathcal{P} u_{n}\right)(s)-(\mathcal{P} u)(s)\right\|= & \left\|\int_{0}^{s} \tilde{\Psi}(s-\sigma, \sigma)\left[f\left(\sigma, u_{n}(\sigma)\right)-f(\sigma, u(\sigma))\right] d \sigma\right\| \\
& +\left\|\int_{0}^{s} \int_{0}^{\sigma} \tilde{\Psi}(s-\sigma, \sigma) \tilde{\Phi}(\sigma, t)\left[f\left(t, u_{n}(t)\right)-f(t, u(t))\right] d t d \sigma\right\|
\end{aligned}
$$




$$
\begin{aligned}
& \quad+\left\|\sum_{0<s_{k}<s} \tilde{\Psi}\left(s_{k}-\sigma, \sigma\right) I_{k}\left(\int_{s_{k}-\tau_{k}}^{s_{k}-\theta_{k}}\left[G\left(t, u_{n}(t)\right)-G(t, u(t))\right] d t\right)\right\| \\
& \leq C \int_{0}^{s}(s-\sigma)^{q-1}\left\|f\left(\sigma, u_{n}(\sigma)\right)-f(\sigma, u(\sigma))\right\| d \sigma \\
& +C^{2} \int_{0}^{s} \int_{0}^{\sigma}(s-\sigma)^{q-1}(\sigma-t)^{p-1}\left\|f\left(t, u_{n}(t)\right)-f(t, u(t))\right\| d t d \sigma \\
& +2 S C L_{N}\left\|u_{n}-u\right\| \sum_{0<s_{k}<s}\left(s_{k}-\sigma\right)^{q-1} D_{k},
\end{aligned}
$$

which gives that, for every $s \in J,\left\|\left(\mathcal{P} u_{n}\right)(s)-(\mathcal{P} u)(s)\right\| \rightarrow 0$ as $n \rightarrow \infty$,

Therefore, $\mathcal{P}: G_{R}: \rightarrow G_{R}$ is a continuous operator. It remains to prove that $\left\{\mathcal{P} u: u \in G_{R}\right\}$ is an equicontinuous function set. For any $u \in S_{R}$ and $s_{1}, s_{2} \in[0, S], s_{1}<s_{2}$, we get

$$
\begin{aligned}
& \|\left(\mathcal{P} u\left(s_{2}\right)-(\mathcal{P} u)\left(s_{1}\right) \|\right. \\
& \leq\left\|\int_{0}^{s_{1}}\left[\tilde{\Psi}\left(s_{2}-\sigma, \sigma\right)-\tilde{\Psi}\left(s_{1}-\sigma, \sigma\right)\right] \tilde{U}(\sigma) \mathbb{A}(0) u_{0} d \sigma\right\| \\
& \quad+\left\|\int_{0}^{s_{1}}\left[\tilde{\Psi}\left(s_{2}-\sigma, \sigma\right)-\tilde{\Psi}\left(s_{1}-\sigma, \sigma\right)\right] f(\sigma, u(\sigma)) d \sigma\right\| \\
& \quad+\left\|\int_{0}^{s_{1}} \int_{0}^{\sigma}\left[\tilde{\Psi}\left(s_{2}-\sigma, \sigma\right)-\tilde{\Psi}\left(s_{1}-\sigma, \sigma\right)\right] \tilde{\Phi}(\sigma, t) f(t, u(t)) d t d \sigma\right\| \\
& \quad+\left\|\int_{s_{1}}^{s_{2}} \tilde{\Psi}\left(s_{2}-\sigma, \sigma\right) \tilde{U}(\sigma) A(0) u_{0} d \sigma\right\| \\
& \quad+\left\|\int_{s_{1}}^{s_{2}} \tilde{\Psi}\left(s_{2}-\sigma, \sigma\right) f(\sigma, u(\sigma)) d \sigma\right\| \\
& \quad+\left\|\int_{s_{1}}^{s_{2}} \int_{0}^{\sigma} \tilde{\Psi}\left(s_{2}-\sigma, \sigma\right) \tilde{\Phi}(\sigma, t) f(t, u(t)) d t d \sigma\right\| \\
& \quad+\left\|\sum_{0<s_{k}<s_{2}-s_{1}} \tilde{\Psi}\left(s_{k}-\sigma, \sigma\right) I_{k}\left(\int_{s_{k}-\tau_{k}}^{s_{k}-\theta_{k}} N(t, u(t)) d t\right)\right\| \\
& \leq I^{1}+I^{2}+I^{3}+I^{4}+I^{5}+I^{6}+I^{t},
\end{aligned}
$$

where

$$
\begin{aligned}
& \left.I^{1}=\int_{0}^{s_{1}} \| \tilde{\Psi}\left(s_{2}-\sigma, \sigma\right)-\tilde{\Psi}\left(s_{1}-\sigma, \sigma\right)\right] \tilde{U}(\sigma) \mathbb{A}(0) u_{0} \| d \sigma \\
& I^{2}=\int_{0}^{s_{1}}\left\|\left[\tilde{\Psi}\left(s_{2}-\sigma, \sigma\right)-\tilde{\Psi}\left(s_{1}-\sigma, \sigma\right)\right]\right\| h_{R}(\sigma) d \sigma \\
& \left.I^{3}=\int_{0}^{s_{1}} \int_{0}^{\sigma} \| \tilde{\Psi}\left(s_{2}-\sigma, \sigma\right)-\tilde{\Psi}\left(s_{1}-\sigma, \sigma\right)\right] \tilde{\Phi}(\sigma, t) \| h_{R}(t) d t d \sigma, \\
& I^{4}=\int_{s_{1}}^{s_{2}}\left\|\tilde{\Psi}\left(s_{2}-\sigma, \sigma\right) \tilde{U}(\sigma) A(0) u_{0}\right\| d \sigma \\
& I^{5}=\int_{s_{1}}^{s_{2}}\left\|\tilde{\Psi}\left(s_{2}-\sigma, \sigma\right)\right\| h_{R}(\sigma) d \sigma, \\
& I^{6}=\int_{s_{1}}^{s_{2}} \int_{0}^{\sigma}\left\|\tilde{\Psi}\left(s_{2}-\sigma, \sigma\right) \tilde{\Phi}(\sigma, t)\right\| h_{R}(t) d t d \sigma, \\
& I^{t}=\sum_{0<s_{k}<s_{2}-s_{1}}\left\|\tilde{\Psi}\left(s_{k}-\sigma, \sigma\right) I_{k}\left(\int_{s_{k}-\tau_{k}}^{s_{k}-\theta_{k}} N(t, u(t)) d t\right)\right\| .
\end{aligned}
$$


Now, we prove that $I^{j} \rightarrow 0$ independently of $u \in S_{R}$ as $s_{2}-s_{1} \rightarrow 0$ for $j=1,2,3,4,5,6, t$.

First, we prove $I^{1}=0$. For $\epsilon>0$ and $s_{1}>0$, by the continuity of $\tilde{\Psi}(s-\sigma, \sigma)$ in the uniform topology in $s$ and $\sigma$, for $0 \leq s \leq S$ and $0 \leq \sigma \leq s-\epsilon$, by Lemma 2.1, we have

$$
\begin{aligned}
I^{1} \leq & \sup _{\sigma \in\left[0, s_{1}-\epsilon\right]}\left\|\tilde{\Psi}\left(s_{2}-\sigma, \sigma\right)-\tilde{\Psi}\left(s_{1}-\sigma, \sigma\right)\right\| \cdot C\left\|\mathbb{A}(0) u_{0}\right\| \int_{0}^{s_{1}-\epsilon}\left(1+\sigma^{p}\right) d \sigma \\
& +C^{2}\left\|\mathbb{A}(0) u_{0}\right\| \int_{s_{1}-\epsilon}^{s_{1}}\left[\left(s_{2}-\sigma\right)^{q-1}-\left(s_{1}-\sigma\right)^{q-1}\right]\left(1+\sigma^{p}\right) d \sigma \\
& \rightarrow 0 \quad \text { as } \epsilon \rightarrow 0 \text { and } s_{2} \rightarrow s_{1} .
\end{aligned}
$$

Obviously, $I^{2}=0$. For $\epsilon>0$ and $s_{1}>0$, using the continuity of $\tilde{\Psi}(s-\sigma, \sigma)$ in the uniform topology in $t$ and $\sigma$, for $0 \leq s \leq S$ and $0 \leq \sigma \leq s-\epsilon$, by Lemma 2.1, we get

$$
\begin{aligned}
I^{2} \leq & \left(s_{1}-\epsilon\right)^{1-q}\left\|h_{R}\right\|_{L^{\frac{1}{q_{1}}}} \sup _{[0, S]}\left\|\tilde{\Psi}\left(s_{2}-\sigma, \sigma\right)-\tilde{\Psi}\left(s_{1}-\sigma, \sigma\right)\right\| \\
& +C \int_{s_{1}-\epsilon}^{s_{1}}\left[\left(s_{2}-\sigma\right)^{q-1}-\left(s_{1}-\sigma\right)^{q-1}\right] h_{R}(\sigma) d \sigma \\
& \rightarrow 0 \quad \text { as } \epsilon \rightarrow 0 \text { and } s_{2} \rightarrow s_{1} .
\end{aligned}
$$

Obviously, $I^{3}=0$, for $s_{1}=0$ and $0<s_{2} \leq S$. For $s_{1}>0$ and $\epsilon>0$, by Lemma 2.1, $\left(F_{1}\right)$, and since the functions $\sigma \rightarrow\left(s_{2}-\sigma\right)^{q-1} I_{\sigma}^{p} h_{R}(\sigma)$ and $\sigma \rightarrow\left(s_{1}-\sigma\right)^{q-1} I_{\sigma}^{p} h_{R}(\sigma)$ are Lebesgue integrable, as well as $\tilde{\Psi}(s-\sigma, \sigma)$ is continuous in the uniform operator topology in $s$ and $\sigma$, for $0 \leq s \leq S$ and $0 \leq \sigma \leq s-\epsilon$, we get

$$
\begin{aligned}
I^{3} \leq & \sup _{\sigma \in\left[0, s_{1}-\epsilon\right]}\left\|\tilde{\Psi}\left(s_{2}-\sigma, \sigma\right)-\tilde{\Psi}\left(s_{1}-\sigma, \sigma\right)\right\| \cdot C \int_{0}^{s_{1}-\epsilon} \int_{0}^{\sigma}(\sigma-t)^{p-1} d t d \sigma \\
& +C^{2} \int_{s_{1}-\epsilon}^{s_{1}} \int_{0}^{\sigma}\left[\left(s_{2}-\sigma\right)^{q-1}-\left(s_{1}-\sigma\right)^{q-1}\right](\sigma-t)^{p-1} h_{R}(t) d t d \sigma \\
\leq & \left(\frac{1-q_{1}}{q-q_{1}}\right)^{1-q_{1}} \frac{C s_{1}^{p}\left\|h_{R}\right\|}{L^{\frac{1}{q_{1}}}[0, S]} \\
& +C^{2} p(p) \int_{s_{1}-\epsilon}^{s_{1}}\left[\left(s_{2}-\sigma\right)^{q-1} I_{\sigma}^{p} h_{R}(\sigma)-\left(s_{1}-\sigma\right)^{q-1} I_{\sigma}^{p} h_{R}(\sigma)\right] d \sigma\left(s_{2}-\sigma, \sigma\right)-\tilde{\Psi}\left(s_{1}-\sigma, \sigma\right) \| \\
& \rightarrow 0 \quad \text { as } \epsilon \rightarrow 0 \text { and } s_{2} \rightarrow s_{1} .
\end{aligned}
$$

For $I^{4}$, by Lemma 2.1, we see that

$$
I^{4} \leq C^{2}\left\|A(0) u_{0}\right\| \int_{s_{1}}^{s_{2}}\left(s_{2}-\sigma\right)^{q-1}\left(1+\sigma^{p}\right) d \sigma \rightarrow 0 \quad \text { as } s_{2} \rightarrow s_{1} .
$$

For $I^{5}$, using Lemma 2.1, $\left(F_{1}\right)$, and Hölder inequality, we have

$$
\begin{aligned}
I^{5} & \leq C \int_{s_{1}}^{s_{2}}\left(s_{2}-\sigma\right)^{q-1} h_{R}(\sigma) d \sigma \\
& \leq C\left(\int_{s_{1}}^{s_{2}}\left(s_{2}-\sigma\right)^{\frac{q-1}{1-q_{1}}}\right)^{1-q_{1}}\left(\int_{s_{1}}^{s_{2}} h_{R}^{\frac{1}{q_{1}}}(\sigma) d \sigma\right)^{q_{1}}
\end{aligned}
$$




$$
\begin{aligned}
& \leq C\left(\frac{1-q_{1}}{q-q_{1}}\right)^{1-q_{1}}\left\|h_{R}\right\|_{L^{\frac{1}{q_{1}}[0, S]}}\left(s_{2}-s_{1}\right)^{q-q_{1}} \\
& \rightarrow 0 \quad \text { as } s_{2} \rightarrow s_{1} .
\end{aligned}
$$

For $I^{6}$, using Lemma 2.1, $\left(F_{1}\right)$, and the fact that the function $\sigma \rightarrow\left(s_{1}-\sigma\right)^{q-1} I_{\sigma}^{p} h_{R}(\sigma)$ is Lebesgue integrable, we get

$$
\begin{aligned}
I^{6} & \leq C^{2} \int_{s_{1}}^{s_{2}} \int_{0}^{\sigma}\left(s_{2}-\sigma\right)^{q-1}(\sigma-t)^{p-1} h_{R}(t) d t d \sigma \\
& \leq C^{2} p(p) \int_{s_{1}}^{s_{2}}\left(s_{2}-\sigma\right)^{q-1} I_{\sigma}^{p} h_{R}(\sigma) d \sigma \\
& \rightarrow 0 \quad \text { as } s_{2} \rightarrow s_{1} .
\end{aligned}
$$

Also $I^{t}=0$ as $s_{2} \rightarrow s_{1}$.

Hence $\left\|(\mathcal{P} u)\left(s_{2}\right)-(\mathcal{P} u)\left(s_{1}\right)\right\|$ tends to 0 independently of $u \in G_{R}$ as $s_{2} \rightarrow s_{1}$, which means that the operator $\mathcal{P}: G_{R} \rightarrow G_{R}$ is equicontinuous.

Let $D=\overline{\mathrm{co}} \mathcal{P}\left(G_{R}\right)$, where $\overline{\text { co }}$ denotes the closure of the convex hull. Then it can be easily seen that the operator $\mathcal{P}: D \rightarrow D(D \subset C(J, F))$ is equicontinuous.

Now, we show that $\mathcal{P}: D \rightarrow D$ is a condensing operator. For any $B \subset D$, by Lemma 2.3, there exists a countable set $B_{0}=\left\{u_{n}\right\} \subset B$ such that

$$
\mu_{C}(\mathcal{P}(B)) \leq 2\left(\mathcal{P}\left(B_{0}\right)\right)
$$

From the equicontinuity of $B, B_{0} \subset B$ is also equicontinuous. Consequently, from Lemma 2.4 and $\left(F_{2}\right)$, we get

$$
\begin{aligned}
\mu\left(\mathcal{P}\left(B_{0}(s)\right)\right)= & \mu\left(u_{0}+\int_{0}^{s} \tilde{\Psi}(s-\sigma, \sigma) \tilde{U}(\sigma) \mathbb{A}(0) u_{0} d \sigma\right) \\
& +\mu\left(\int_{0}^{s} \tilde{\Psi}(s-\sigma, \sigma) f\left(\sigma, u_{n}(\sigma)\right) d \sigma\right) \\
& +\mu\left(\int_{0}^{s} \int_{0}^{\sigma} \tilde{\Psi}(s-\sigma, \sigma) \tilde{\Phi}(\sigma, t) f\left(t, u_{n}(t)\right) d t d \sigma\right) \\
& +\mu\left(\sum_{0<s_{k}<s} \tilde{\Psi}\left(s_{k}-\sigma, \sigma\right) I_{k}\left(\int_{s_{k}-\tau_{k}}^{s_{k}-\theta_{k}}\left(N, u_{n}(t)\right) d t\right)\right) \\
\leq & 2 C \int_{0}^{s}(s-\sigma)^{q-1} \mu\left(f\left(\sigma, u_{n}(\sigma)\right)\right) d \sigma \\
& +4 C^{2} \int_{0}^{s} \int_{0}^{\sigma}(s-\sigma)^{q-1}(\sigma-t)^{p-1} \mu\left(f\left(t, u_{n}(t)\right)\right) d t d \sigma \\
& +2 C\left(\sum_{0<s_{k}<s}(s-\sigma)^{q-1} I_{k}\left(\int_{s_{k}-\tau_{k}}^{s_{k}-\theta_{k}} \mu\left(N, u_{n}(t)\right) d t\right)\right) \\
\leq & 2 C \int_{0}^{s}(s-\sigma)^{q-1} L \mu\left(B_{0}(\sigma)\right) d \sigma \\
& +4 C^{2} \int_{0}^{s} \int_{0}^{\sigma}(s-\sigma)^{q-1}(\sigma-t)^{p-1} L \mu\left(B_{0}(t)\right) d t d \sigma
\end{aligned}
$$




$$
\begin{aligned}
& +2 C\left(\sum_{0<s_{k}<s}(s-\sigma)^{q-1} I_{k}\left(\int_{s_{k}-\tau_{k}}^{s_{k}-\theta_{k}} L_{N} \mu\left(B_{0}(t)\right) d t\right)\right) \\
\leq & 2 C L \int_{0}^{s}(s-\sigma)^{q-1} d \sigma \mu_{C}(B) \\
& +4 C^{2} L \mathbf{B}(q, p) \int_{0}^{s}(s-\sigma)^{q+p-1} d \sigma \mu_{C}(B) \\
& +2 C L_{N}\left(\sum_{0<s_{k}<s}(s-\sigma)^{q-1} I_{k}\left(\int_{s_{k}-\tau_{k}}^{s_{k}-\theta_{k}} \mu_{C}(B) d s\right)\right) \\
\leq & 2 C S^{q}\left[\frac{L}{q}+\frac{2 C L \mathbf{B}(q, p) T^{p}}{q+p}+2 L_{N} S\right] \mu_{C}(B) .
\end{aligned}
$$

From Lemma 2.5 and since $\mathcal{P}\left(B_{0}\right) \subset B$ is bounded and equicontinuous, we have

$$
\mu_{C}\left(\mathcal{P}\left(B_{0}\right)\right)=\max _{s \in[0, S]} \mu\left(\mathcal{P}\left(B_{0}\right)(s)\right)
$$

Therefore, by (2.5)-(2.6), we have

$$
\mu_{C}(\mathcal{P}(B)) \leq 4 C S^{q}\left[\frac{L}{q}+\frac{2 C L \mathbf{B}(q, p) T^{p}}{q+p}+2 L_{N} S\right] \mu_{C}(B) .
$$

By combining (3.10), (3.4) and Definition 2.5, we know that $\mathcal{P}: G_{R} \rightarrow G_{R}$ is a $k$-set contractive operator. By Lemma 2.6, $\mathcal{P}$ has at least one fixed point $u \in G_{R}$. Therefore $\mathcal{P}$ is a mild solution of (1.1)-(1.3).

\section{Example}

Consider the following nonlinear reaction-diffusion equation with integral impulse condition:

$$
\begin{aligned}
& { }^{C} D^{q} u(z, s)-b(s) \Delta u(z, s)=f(z, s, u(z, s)), \\
& u(z, s)=0, \quad z \in \partial G, s \in J, \\
& \Delta^{*} u\left(z, s_{1}\right)=I_{1}\left(\int_{s_{1}-\tau_{1}}^{s_{1}-\theta_{1}} N(z, t, u(z, t)) d t\right),
\end{aligned}
$$

where ${ }^{C} D^{q}$ is a CFD of order $0<q \leq 1, b(s)$ is diffusion coefficient which is continuous on $J=[0, S]$, and $\left|b\left(s_{2}\right)-b\left(s_{1}\right)\right| \leq C\left|s_{2}-s_{1}\right|^{p}, s_{1}, s_{2} \in J, 0<p \leq 1, C$ is a positive constant independent of $s_{1}$ and $s_{2}, \Delta$ is Laplace operator; $S \subset \mathbb{R}^{m}$ is a bounded domain with a sufficiently smooth boundary $\partial G ; f, N: G \times J \times \mathbb{R} \rightarrow \mathbb{R}$ are nonlinear functions.

Let $\|\cdot\|$ denote the $L^{2}$-norm on the Banach space $F=L^{2}(G)$. In $F$ we define a linear operator by

$$
\mathbb{A}(s) u=b(s) \Delta u
$$

with the domain

$$
D(A)=H^{2}(G) \cap H_{0}^{1}(G),
$$


where $H^{2}(G)$ is the completion of $C^{2}(G)$ with respect to the norm

$$
\|u\|_{H^{2}(G)}=\left(\int_{G} \sum_{|\nu| \leq 2}\left|D^{v} u(z)\right|^{2} d z\right)^{\frac{1}{2}},
$$

$H_{0}^{1}(G)$ is the completion of $C^{1}(G)$ with respect to the norm $\|u\|_{H^{1}(G)}$, and $C_{0}^{1}(G)$ is the set of all functions $u \in C^{1}(G)$ with compact support on the domain $G$. From [34], we know that $-\mathbb{A}(t)$ generates an analytic semigroup $e^{-s \mathbb{A}(t)}$ in $E$ satisfying $\left(H_{3}\right)$ and $\left(H_{4}\right)$. Let $u(s)=u(\cdot, s)$, $f(s, u(s))=f(\cdot, s, u(\cdot, s)), N(s, u(s))=N(\cdot, s, u(\cdot, s))$. Then system (4.1)-(4.3) can be modified into system (1.1)-(1.3).

Let the function $f$ satisfy the following condition:

(i) There is a bounded function $h_{r}(s)$ such that for any $s \in[0, S], z \in G$, and $u \in L^{2}(G)$ satisfying $\left(\int_{G}|u(z)|^{2} d z\right)^{\frac{1}{2}} \leq r$ for some $r>0$,

$$
\left(\int_{G}|f(s, u(z, s))|^{2} d z\right)^{\frac{1}{2}} \leq h_{r}(s) .
$$

Theorem 4.1 Consider the nonlinear function $f(z, s, u(z, s))=\frac{\cos (z, s, u(z, s))}{e^{s}}$ and the function $I_{1}: \mathbb{R} \rightarrow \mathbb{R}$ defined by $I_{1}(z)=\cos z$. Then for the choices $\theta_{1}=0, \tau_{1}=1$, problem (4.1)-(4.3) has at least one mild solution.

Proof: By the definition of $f$, condition $\left(F_{1}\right)$ is clearly satisfied. Also $I_{1}$ and $N$ are Lipschitz functions. Condition (3.4) is satisfied with $q=\frac{1}{2}$ and $0 \leq q_{1}<\frac{1}{2}, h_{r}(s)=\frac{\sqrt{\operatorname{meas}(G)}}{e^{s}}$, $\rho=0$. So all the assumptions of Theorem 3.4 are satisfied. Hence the initial boundary value problem to the nonlinear reaction-diffusion equation with integral impulse condition has at least one mild solution due to Theorem 3.1.

\section{Conclusions}

The existence of solutions of non-autonomous fractional differential equations with integral impulse condition by using the measure of non-compactness has been discussed in this article. One can extend this work for impulsive non-autonomous fractional differential equations with integral impulse condition by using the fixed point theorems.

Funding

Not applicable.

Availability of data and materials

Not applicable.

Competing interests

The authors declare that they have no competing interests.

Authors' contributions

The authors contributed equally and significantly in writing this paper. All authors read and approved the final manuscript.

\section{Author details}

'Department of Mathematics, Government PG College, Bina, Sagar 470113, Madhya Pradesh, India. ${ }^{2}$ Department of Mathematics, University of Delhi, Delhi 110007, India. ${ }^{3}$ Post Graduate and Research Department of Mathematics, Kongunadu Arts and Science College (Autonomous), Coimbatore 641 029, Tamil Nadu, India. ${ }^{4}$ Department of Mathematics, College of Arts and Sciences, Prince Sattam bin Abdulaziz University, Wadi Aldawasir 11991, Saudi Arabia. ${ }^{5}$ Department of Mathematics, Cankaya University, Ankara, Turkey. ${ }^{6}$ Institute of Space Sciences, Magurele-Bucharest, Romania. ${ }^{7}$ Department of Medical Research, China Medical University Hospital, China Medical University, Taichung, Taiwan. 


\section{Publisher's Note}

Springer Nature remains neutral with regard to jurisdictional claims in published maps and institutional affiliations.

\section{Received: 23 April 2020 Accepted: 9 August 2020 Published online: 20 August 2020}

\section{References}

1. Agrawal, O.P.: Solution for a fractional diffusion-wave equation defined in a bounded domain. Nonlinear Dyn. 29(1-4), 145-155 (2002)

2. Banas, J.: On measures of noncompactness in Banach spaces. Comment. Math. Univ. Carol. 21(1), 131-143 (1980)

3. Banas, J., Goebel, K.: Measure of Noncompactness in Banach Spaces. Lecture Notes in Pure and Appl. Math. Dekker, New York (1980)

4. Benchohra, M., Litimein, S., Nieto, J..: Semilinear fractional differential equations with infinite delay and non-instantaneous impulses. J. Fixed Point Theory Appl. 21(1), 21 (2019)

5. Chen, P., Zhang, X:: Approximate controllability of nonlocal problem for non-autonomous stochastic evolution equations. Evol. Equ. Control Theory (2020). https://doi.org/10.3934/eect.2020076

6. Chen, P., Zhang, X., Li, Y.: Mixed monotone iterative technique for a class of semilinear impulsive evolution equations in Banach spaces. Nonlinear Anal., Theory Methods Appl. 74(11), 3578-3588 (2011)

7. Chen, P., Zhang, X., Li, Y.: Existence of mild solutions to partial differential equations with non-instantaneous impulses. Electron. J. Differ. Equ. 2016, 241 (2016)

8. Chen, P., Zhang, X., Li, Y.: Cauchy problem for stochastic non-autonomous evolution equations governed by noncompact evolution families. Discrete Contin. Dyn. Syst., Ser. B (2020). https://doi.org/10.3934/dcdsb.2020171

9. Chen, P., Zhang, X., Li, Y.: Iterative method for a new class of evolution equations with non-instantaneous impulses. Taiwan. J. Math. 21(4), 913-942 (2017)

10. Chen, P., Zhang, X., Li, Y.: Study on fractional non-autonomous evolution equations with delay. Comput. Math. Appl. 73(5), 794-803 (2017)

11. Chen, P., Zhang, X., Li, Y.: A blowup alternative result for fractional nonautonomous evolution equation of Volterra type. Commun. Pure Appl. Anal. 17(5), 1975-1992 (2018)

12. Chen, P., Zhang, X., Li, Y.: Fractional non-autonomous evolution equation with nonlocal conditions. J. Pseudo-Differ. Oper. Appl. 10(4), 955-973 (2019)

13. Chen, P., Zhang, X., Li, Y.: Non-autonomous evolution equations of parabolic type with non-instantaneous impulses. Mediterr. J. Math. 16(5), 118 (2019)

14. Chen, P., Zhang, X., Li, Y.: Approximate controllability of non-autonomous evolution system with nonlocal conditions. J. Dyn. Control Syst. 26(1), 1-16 (2020)

15. Chen, P., Zhang, X., Li, Y.: Cauchy problem for fractional non-autonomous evolution equations. Banach J. Math. Anal. 14(2), 559-584 (2020)

16. Chen, P., Zhang, X., Li, Y.: Existence and approximate controllability of fractional evolution equations with nonlocal conditions via resolvent operators. Fract. Calc. Appl. Anal. 23(1), 268-291 (2020)

17. Daftardar-Gejji, V., Jafari, H.: Analysis of a system of nonautonomous fractional differential equations involving Caputo derivatives. J. Math. Anal. Appl. 328(2), 1026-1033 (2007)

18. El-Borai, M.M.: The fundamental solutions for fractional evolution equations of parabolic type. J. Appl. Math. Stoch. Anal. 2004(3), 197-212 (2004)

19. Francesco, M.: Fractional Calculus and Waves in Linear Viscoelasticity. An Introduction to Mathematical Models. World Scientific, Singapore (2010)

20. Hernandez, E., O'Regan, D.: Existence of solutions for abstract non-autonomous neutral differential equations. Can. Math. Bull. 55(4), 736-751 (2012)

21. Kavitha, K., Vijayakumar, V., Udhayakumar, R.: Results on controllability of Hilfer fractional neutral differential equations with infinite delay via measures of noncompactness. Chaos Solitons Fractals 139, 110035 (2020). https://doi.org/10.1016/j.chaos.2020.110035

22. Kilbas, A.A., Srivastava, H.M., Trujillo, J.J.: Theory and Applications of Fractional Differential Equations. North-Holland Mathematics Studies. Elsevier, New York (2006)

23. Kucche, K.D., Shikhare, P.U.: On impulsive delay integrodifferential equations with integral impulses. Mediterr. J. Math. $17,103(2020)$

24. Kumar, S., Kumar, A., Odibat, Z., Aldhaifallah, M., Nisar, K.S.: A comparison study of two modified analytical approach for the solution of nonlinear fractional shallow water equations in fluid flow. AIMS Math. 5(4), 3035-3055 (2020)

25. Li, F: Solvability of nonautonomous fractional integrodifferential equations with infinite delay. Adv. Differ. Equ. 2011(4), 806729 (2011)

26. Logeswari, K., Ravichandran, C.: A new exploration on existence of fractional neutral integro-differential equations in the concept of Atangana-Baleanu derivative. Physica A 544, 123454 (2020)

27. Mainardi, F.: Fractional relaxation-oscillation and fractional diffusion-wave phenomena. Chaos Solitons Fractals 7(9), 1461-1477 (1996)

28. Malik, M., Dhayal, R., Abbas, S.: Controllability of non-autonomous nonlinear differential system with non-instantaneous impulses. Rev. R. Acad. Cienc. Exactas Fís. Nat., Ser. A Mat. 113(1), 103-118 (2019)

29. Meraj, A., Narain, D.: Existence and uniqueness of extremal mild solutions for non-autonomous nonlocal integro-differential equations via monotone iterative technique. Filomat 33(10), 2985-2993 (2019)

30. Miller, K.S., Ross, B.: An Introduction to the Fractional Calculus and Differential Equations. Wiley, New York (1993)

31. Monch, H.: Boundary value problems for nonlinear ordinary differential equations of second order in Banach spaces. Nonlinear Anal., Theory Methods Appl. 4(5), 985-999 (1980)

32. Nisar, K.S., Shaikh, A., Rahman, G., Kumar, D.: Solution of fractional kinetic equations involving class of functions and Sumudu transform. Adv. Differ. Equ. 2020(1), 39 (2020)

33. Panda, S.K., Abdeljawad, T., Ravichandran, C.: A complex valued approach to the solutions of Riemann-Liouville integral, Atangana-Baleanu integral operator and non-linear telegraph equation via fixed point method. Chaos Solitons Fractals 130, 109439 (2020) 
34. Pazy, A.: Semigroups of Linear Operators and Applications to Partial Differential Equations. Springer, Berlin (2012)

35. Pierri, M., O’Regan, D.: On non-autonomous abstract nonlinear fractional differential equations. Appl. Anal. 94(5), 879-890 (2015)

36. Podlubny, l.: An introduction to fractional derivatives, fractional differential equations, to methods of their solution and some of their applications. Math. Sci. Eng., 198, xxiv+340 (1999)

37. Ravichandran, C., Logeswari, K., Jarad, F.: New results on existence in the framework of Atangana-Baleanu derivative for fractional integro-differential equations. Chaos Solitons Fractals 125, 194-200 (2019)

38. Ravichandran, C., Valliammal, N., Nieto, J.J.: New results on exact controllability of a class of neutral integrodifferential systems with state dependent delay in Banach spaces. J. Franklin Inst. 356(3), 1535-1565 (2019)

39. Richard, H.: Fractional Calculus: An Introduction for Physicists. World Scientific, Singapore (2014)

40. Tassaddiq, A., Khan, I., Nisar, K.S.: Heat transfer analysis in sodium alginate based nanofluid using MoS $_{2}$ nanoparticles: Atangana-Baleanu fractional model. Chaos Solitons Fractals 130, 109445 (2020)

41. Valliammal, N., Ravichandran, C., Park, J.H.: On the controllability of fractional neutral integrodifferential delay equations with nonlocal conditions. Math. Methods Appl. Sci. 40(14), 5044-5055 (2017)

42. Vijayakumar, V., Udhayakumar, R.: Results on approximate controllability for non-densely defined Hilfer fractional differential system with infinite delay. Chaos Solitons Fractals 139, 110019 (2020). https://doi.org/10.1016/j.chaos.2020.110019

43. Wang, J., Lin, Z.: A class of impulsive nonautonomous differential equations and Ulam-Hyers-Rassias stability. Math Methods Appl. Sci. 38, 868-880 (2015). Communicated by Lachowicz, M.A.

44. Zhang, X., Chen, P., Li, Y.: Fractional retarded differential equations involving mixed nonlocal plus local initial conditions. Numer. Funct. Anal. Optim. 40(14), 1678-1702 (2019)

45. Zhang, X., Li, Y., Chen, P.: Existence of extremal mild solutions for the initial value problem of evolution equations with non-instantaneous impulses. J. Fixed Point Theory Appl. 19(4), 3013-3027 (2017)

\section{Submit your manuscript to a SpringerOpen ${ }^{\circ}$ journal and benefit from:}

- Convenient online submission

- Rigorous peer review

- Open access: articles freely available online

- High visibility within the field

Retaining the copyright to your article

Submit your next manuscript at springeropen.com 